PROCEEDINGS OF THE AMERICAN MATHEMATICAL SOCIETY

Volume 125, Number 4, April 1997, Pages 1019-1026

S 0002-9939(97)03555-7

\title{
LARGE TIME BEHAVIOR FOR DISCONTINUOUS DYNAMICS ON HILBERT SPACES
}

\author{
VÍCTOR H. CORTÉS AND HUMBERTO PRADO
}

(Communicated by Palle E. T. Jorgensen)

\begin{abstract}
This paper is concerned with the behavior in time for a certain class of dynamics which are discontinuous with respect to the time variable. We introduce the corresponding wave operators and we ensure their existence. Moreover, under suitable conditions this class of wave operators can be approximated in the strong sense by a sequence of ordinary wave operators. Our results can be applied to impulsive dynamical systems.
\end{abstract}

\section{INTRODUCTION}

We study the asymptotic behavior as $t \rightarrow \pm \infty$ of certain families of operators $U(t)$ acting on Hilbert spaces; these families are piecewise strongly continuous and they do not form a group nor a semigroup. Then we analyze the problem of the asymptotic comparison of a unitary group $e^{-i t H}$ with a discontinuous family $U(t)$. Within this setting we introduce the corresponding wave operators. We also discuss several examples. Moreover, we notice that under suitable conditions, this class of wave operators can be approximated in the strong operator norm by a sequence of ordinary wave operators associated to unitary groups.

We now introduce our general framework: Let $\tau=\left\{\alpha_{n}\right\}$ be an increasing sequence of real numbers such that $\lim \alpha_{n}=\infty$ as $n \rightarrow \pm \infty$. Suppose that $U=\{U(t, s): s, t \in \mathbb{R}\}$ is a two-parameter family of bounded operators acting on a Hilbert space $\mathcal{H}$ such that the bounded operator-valued functions $t \mapsto U(t, s)$ are strongly continuous for each $s \in \mathbb{R}$, and for each $t \in \mathbb{R}$ the functions $s \mapsto U(t, s)$ are piecewise strongly continuous on the intervals $\alpha_{n} \leq s<\alpha_{n+1}$. Then we call the triple $(\mathcal{H}, U, \tau)$ an impulsive system, and the family $U(t, s)$ is called an impulsive dynamics. If there is no confusion we simply denote an impulsive system by $U(t, s)$.

In the succeeding sections we consider the problem of the asymptotic comparison of an impulsive dynamics $U(t, s)$ with a one-parameter unitary group of operators $U_{0}(t)=e^{-i t H_{0}}$, both acting on the same Hilbert space $\mathcal{H}$. Specifically, let $(\mathcal{H}, U, \tau)$ be an impulsive system and let $\tau$ be an infinite sequence of real numbers defined by the partition $\cdots<\alpha_{-n}<\cdots<\alpha_{1}<\cdots<\alpha_{n}<\alpha_{n+1}<\cdots$. Let $p$ be a step function such that $p(t)=p\left(\alpha_{n}\right)$ whenever $\alpha_{n} \leq t<\alpha_{n+1}$. Then, in the next sections we present the necessary assumptions needed to ensure the existence of the

Received by the editors July 14, 1995.

1991 Mathematics Subject Classification. Primary 47A40, 47B48, 47A53, 47A58.

This research has been partially supported by FONDECYT through grant 1930023, and UCN 40340209 . 
strong limits

$$
W_{ \pm}=s-\lim _{t \rightarrow \pm \infty} U^{*}(t, p(t)) U_{0}(t)
$$

where $U^{*}(t, p(t))$ denotes the Hilbert adjoint of $U(t, p(t))$. If the limits exist, we call the operators $W_{ \pm}$the impulsive wave operators.

As an example of impulsive dynamics, let us suppose that $\tau=\left\{\alpha_{n}\right\}$ is an increasing and unbounded sequence of real numbers. For each $x \in \mathbb{R}^{n}$, we let $V(; x): \mathbb{R} \rightarrow$ $\mathbb{R}$ be a function which is piecewise constant on every interval $\left[\alpha_{n}, \alpha_{n+1}[\right.$, that is, $V(s ; x)=V\left(\alpha_{n} ; x\right)$ for $\alpha_{n} \leq s<\alpha_{n+1}$. Let $M(s)$ be the operator of multiplication by the function $V(s, x)$, acting on the Hilbert space $\mathcal{H}=L^{2}\left(\mathbb{R}^{n}\right)$. Now, suppose that $e^{-i t H}$ is a one-parameter unitary group of operators defined on $\mathcal{H}$, then the two-parameter family $U(t, s)=M(s) e^{-i t H}$ defines an impulsive dynamics on the space $\mathcal{H}$.

In the time dependent approach to quantum mechanical scattering the existence of the wave operators

$$
\Omega_{ \pm}\left(H, H_{0}\right)=s-\lim _{t \rightarrow \pm \infty} e^{i t H} e^{-i t H_{0}}
$$

is established on $L^{2}\left(\mathbb{R}^{n}\right)$ where $H_{0}=-\Delta$ is the usual extension of the Laplacian, $H=H_{0}+V(x)$ is a self-adjoint perturbation of $H_{0}$, and the operator $V(x)$ depends only on the space variable $x \in \mathbb{R}^{n}$, and it is independent of $t$. However, in connection with potential scattering and also in homogeneous (or time dependent) problems time dependent perturbations $V(x, t)$ occur ([5], [7]). In our examples below, we will see that the perturbations to be considered may also depend on the time variable.

Since the impulsive dynamics are only piecewise continuous, we need to implement a modified version of Cook's method from the classical theory of scattering to ensure the existence of the impulsive wave operators $W_{ \pm}$defined via the strong limit (1). Moreover, the necessary assumptions needed can be stated in a general context which is formulated by the following lemma.

Lemma 1 (Generalization of Cook's method). Let $\mathcal{B}$ be a Banach space and let $h$ be a continuous vector-valued function, $h: \mathbb{R} \times \mathbb{R} \rightarrow \mathcal{B}$. Suppose that

(a) $\left\{\alpha_{n}\right\}$ is a strictly increasing sequence of real numbers such that $\inf \left\{\left(\alpha_{n+1}-\right.\right.$ $\left.\left.\alpha_{n}\right): n=0, \pm 1, \pm 2, \ldots\right\}=\delta>0$.

(b) Let $p: \mathbb{R} \rightarrow \mathbb{R}$ be a step function constant on every interval $\left[\alpha_{n}, \alpha_{n+1}[\right.$, such that $p(t) \rightarrow \pm \infty$, as $t \rightarrow \pm \infty$, and $p(t) \leq t$ for all $t$.

(c) The first derivative $D_{1} h$ of $h$, with respect to the $t$ variable exists for all $(t, s)$ and the function $(t, s) \rightarrow\left\|D_{1} h(t, p(s))\right\|$ is in $L^{1}\left(\left[t_{0},+\infty\right) \times\left[s_{0},+\infty\right)\right)$, for some $t_{0}, s_{0} \in \mathbb{R}$.

(d) The infinite series $\sum_{k}\left\|h\left(p\left(\alpha_{k}\right), p\left(\alpha_{k}\right)\right)-h\left(p\left(\alpha_{k}\right), p\left(\alpha_{k-1}\right)\right)\right\|$ converges.

Then the limit, $\lim _{t \rightarrow \pm \infty} h(t, p(t))$, exists in $\mathcal{B}$.

Proof. Since $p$ is a piecewise constant function we denote $p(t)=\lambda(k)$ for $\alpha_{k} \leq$ $t<\alpha_{k+1}$. Notice that $\lambda(k)=p\left(\alpha_{k}\right)=p(t) \leq t$ on each interval $\left[\alpha_{k}, \alpha_{k+1}[\right.$. Now, suppose that $s<t$. We claim that $\|h(t, p(t))-h(s, p(s))\|$ converges to zero as $s \rightarrow+\infty$. In fact,

$$
\begin{aligned}
\| h(t, p(t)) & -h(s, p(s))\|\leq\| h(t, p(t))-h(p(t), p(t)) \| \\
& +\|h(s, p(s))-h(p(s), p(s))\|+\| h(p(t), p(t)-h(p(s), p(s)) \| .
\end{aligned}
$$


Since $D_{1} h$ exists, then, whenever $t \in\left[\alpha_{k}, \alpha_{k+1}[\right.$,

$$
\begin{aligned}
& \|h(t, p(t))-h(p(t), p(t))\|=\|h(t, \lambda(k))-h(\lambda(k), \lambda(k))\| \\
& \quad \leq \int_{\lambda(k)}^{t}\left\|D_{1} h(\tau, p(\tau))\right\| d \tau \leq \int_{\lambda(k)}^{\alpha_{k+1}}\left\|D_{1} h\left(\xi, p\left(\alpha_{m}\right)\right)\right\| d \xi \\
& \quad \leq \frac{1}{\delta} \int_{\alpha_{k}}^{\alpha_{k+1}} \int_{\lambda(k)}^{\alpha_{k+1}}\left\|D_{1} h(\xi, p(\mu))\right\| d \xi d \mu \leq \frac{1}{\delta} \int_{r_{0}}^{+\infty} \int_{r_{1}}^{+\infty}\left\|D_{1} h(\xi, p(\mu))\right\| d \xi d \mu
\end{aligned}
$$

for all integers $k$ such that $r_{0} \leq \alpha_{k}$, and $r_{1} \leq \lambda(k)$. Now suppose $s, t$, and $m$ are sufficiently large such that $\alpha_{m} \leq s<\alpha_{m+1}<\cdots<\alpha_{m+q} \leq t<\alpha_{m+q+1}$. Then we define

$$
I=\sum_{k=1}^{q}\|h(\lambda(m+k), \lambda(m+k-1))-h(\lambda(m+k-1), \lambda(m+k-1))\|
$$

On the other hand,

$$
\begin{aligned}
& \int_{\lambda(m+k-1)}^{\lambda(m+k)}\left\|D_{1} h(\lambda(\tau, \lambda(m+k-1)))\right\| d \tau \\
& \quad \leq \frac{1}{\delta} \int_{\alpha_{m+k+1}}^{\alpha_{m+k}} \int_{\lambda(m+k-1)}^{\lambda(m+k)}\left\|D_{1} h(\tau, p(\mu))\right\| d \tau d \mu \\
& \quad \leq \frac{1}{\delta} \int_{r_{0}}^{+\infty} \int_{r_{1}}^{+\infty}\left\|D_{1} h(\tau, p(\mu))\right\| d \tau d \mu
\end{aligned}
$$

for all $k=m, m+1, \ldots, m+q$, and $r_{0} \leq \alpha_{m+k-1}, r_{1} \leq \lambda(m+k-1)$. Thus, the expression $I$ is bounded above by

$$
\frac{q}{\delta} \int_{r_{0}}^{+\infty} \int_{r_{1}}^{+\infty}\left\|D_{1} h(\tau, p(\mu))\right\| d \tau d \mu
$$

Since $p(t) \rightarrow+\infty$ we can choose $m$ so that $p\left(\alpha_{m}\right)=\lambda(m)$ is sufficiently large. Hence for $\varepsilon>0$, there are $r_{0}, r_{1}$ and $m$ large enough so that $r_{1} \leq \lambda(m)$ and $r_{0} \leq \alpha_{m}$ such that $r_{0} \leq \alpha_{m} \leq s<\alpha_{m+1}<\cdots<\alpha_{m+q} \leq t<\alpha_{m+q+1}$. Then, from hypothesis (c) it follows that the left-hand side of (4) is less than $\varepsilon / 3$. Next we define

$$
S=\sum_{k=1}^{q}\|h(\lambda(m+k), \lambda(m+k))-h(\lambda(m+k), \lambda(m+k-1))\| .
$$

Thus by assumption (d) we have for every $\varepsilon>0$ that there is an integer $m$ such that

$$
S \leq \sum_{k=m+1}^{\infty}\|h(\lambda(k), \lambda(k))-h(\lambda(k), \lambda(k-1))\|<\frac{\varepsilon}{3} .
$$

By choosing appropriate $m$ and $q$, from (4) and (5) we obtain

$$
\begin{aligned}
\|h(p(t), p(t))-h(p(s), p(s))\| & =\|h(\lambda(m+q), \lambda(m+q))-h(\lambda(m), \lambda(m))\| \\
& \leq I+S<2 \frac{\varepsilon}{3} .
\end{aligned}
$$

Thus for each $\varepsilon>0$ we can find an integer $m$ such that each of the left-hand side terms of inequality (3) is less than $\varepsilon / 3$. Therefore, for $s<t, \| h(t, p(t))-$ $h(s, p(s)) \|$ goes to zero as $s \rightarrow \infty$. 
1.1. Remark. We recall that in Quantum Scattering the asymptotic comparison of two different dynamics $e^{-i t H}$ and $e^{-i t H_{0}}$ for the same system, is determined if

$$
s-\lim _{t \rightarrow \pm \infty}\left(e^{-i t H}-e^{-i t H_{0}}\right)=0
$$

which is equivalent to the question about the existence of the strong limits

$$
\Omega_{ \pm}\left(H, H_{0}\right)=s-\lim _{t \rightarrow \pm \infty} e^{i t H} e^{-i t H_{0}} .
$$

In our context we also consider the following type of dynamics: Suppose that $H_{0}$ is a self-adjoint operator on a Hilbert space, let $\tau=\left\{\alpha_{n}\right\}$ be a sequence of real numbers so that $\alpha_{n}<\alpha_{n+1}$ for every integer $n$. Let $V(s)$ be a one-parameter family of operators acting on a Hilbert space $\mathcal{H}$ and such that $V(s)=V\left(\alpha_{n}\right)$ for $s \in\left[\alpha_{n}, \alpha_{n+1}\left[\right.\right.$. Suppose that $H(s)=H_{0}+V(s)$ are self-adjoint for all $s \in \mathbb{R}$. Then by Stone's Theorem, each of the operators $H(s)$ generates a one-parameter unitary group of operators $U(t, s)=e^{-i t H(s)}, s \in \mathbb{R}$. Now letting $s=t$ we denote $U(t)=e^{-i t H(t)}$ then $U(t)$ is no longer a group nor a semigroup. Moreover, the family $U(t)$ is piecewise strongly continuous on every interval $\left[\alpha_{n}, \alpha_{n+1}[\right.$ and with bounded jumps at each $\alpha_{n}$, that is, $s-\lim _{\varepsilon \rightarrow 0^{+}} U\left(\alpha_{n}+\varepsilon\right)=U\left(\alpha_{n}\right)$ and $s-$ $\lim _{\varepsilon \rightarrow 0^{+}} U\left(\alpha_{n}-\varepsilon\right)=e^{-i \alpha_{n} H\left(\alpha_{n-1}\right)}$. Thus according to definition (1) the impulsive wave operators $W_{ \pm}$associated to $e^{i t H(t)} e^{-i t H_{0}}$ are determined by existence of the strong limits

$$
W_{ \pm}=s-\lim _{t \rightarrow \pm \infty} e^{i t H(t)} e^{-i t H_{0}} .
$$

Notice that the jumps of the operator-valued function $U(t)=e^{i t H(t)}$ are given at each $\alpha_{n}$ by

$$
J\left(\alpha_{n}\right)=e^{-i \alpha_{n} H\left(\alpha_{n}\right)}-e^{-i \alpha_{n} H\left(\alpha_{n-1}\right)} .
$$

We call $J\left(\alpha_{n}\right)$ the jump operator at the point $\alpha_{n}$.

We now let $H(t)=H_{0}+V(t)$ be a family of self-adjoint operators for $t$ real. Then the following theorem and its corollary show the existence of the impulsive wave operators $W_{ \pm}=s-\lim _{t \rightarrow \pm \infty} e^{i t H([t])} e^{-i t H_{0}}$, where $[t]$ denotes the integral part of $t$. Furthermore, this class of wave operators can be obtained as a strong limit of a sequence $\Omega_{ \pm}\left(H_{n}, H_{0}\right)=s-\lim _{t \rightarrow \pm \infty} e^{i t H_{n}} e^{-i t H_{0}}$, where $H_{n}=H_{0}+V_{n}$ is a sequence of self-adjoint perturbations of $H_{0}$, such that $\operatorname{Dom}\left(H_{0}\right)=\operatorname{Dom}\left(H_{n}\right)=\operatorname{Dom}\left(V_{n}\right)$ for all integers $n$, and $H_{n}=H([t])$ for all $t$, such that $n \leq t<n+1$. Throughout the remainder of the paper ordinary wave operators are denoted by $\Omega_{ \pm}\left(H, H_{0}\right)$.

Theorem 2. Let $H_{n}$ and $V_{n}$ be sequences of operators satisfying the above assumptions, and assume that $H=H_{0}+V$ is self-adjoint. Suppose

(a) The wave operators $\Omega_{ \pm}\left(H, H_{0}\right)$ and $\Omega_{ \pm}\left(H_{n}, H_{0}\right)$ exist for all integers $n$.

(b) There exists $V$ such that $s-\lim _{n \rightarrow \pm \infty} V_{n}=V$, with $\operatorname{Dom}(V)=\operatorname{Dom}\left(V_{n}\right)$.

(c) The series $S(t)=\sum_{k}\left\|\left(e^{i t H_{k+1}}-e^{i t H_{k}}\right) e^{-i t H_{0}} \phi\right\|$ is uniformly convergent, for $|t|$ sufficiently large and $\lim _{t \rightarrow \pm \infty} S(t)=0$.

Then $W_{ \pm}$exist, and $W_{ \pm}=\Omega_{ \pm}\left(H, H_{0}\right)$.

Proof. Since $s-\lim _{n \rightarrow \infty} V_{n}=V$ on $\operatorname{dom}(V)$, for arbitrary $\phi$ in $\mathcal{H}$ we obtain

$$
\begin{aligned}
\left\|\left(i-H_{n}\right)^{-1} \phi-(i-H)^{-1} \phi\right\| & =\left\|\left(i-H_{n}\right)^{-1}\left(H_{n}-H\right)(i-H)^{-1} \phi\right\| \\
& \leq\left\|\left(V_{n}-V\right)(i-H)^{-1} \phi\right\| .
\end{aligned}
$$


Thus $H_{n}$ converges to $H$ in the strong resolvent sense. Therefore, for each $t$

$$
s-\lim _{n \rightarrow \infty} e^{i t H_{n}} e^{-i t H_{0}}=e^{i t H} e^{-i t H_{0}} .
$$

Now let $\phi_{t}=e^{-i t H_{0}} \phi$, for $\phi \in \mathcal{H}$. Then we have

$$
\left\|\left(e^{i t H([t])}-e^{i t H_{k}}\right) \phi_{t}\right\| \leq \sum_{k}\left\|\left(e^{i t H_{k+1}}-e^{i t H_{k}}\right) \phi_{t}\right\| .
$$

By hypothesis the right-hand side of the above inequality is uniformly convergent and the series as a function of $t$ converges to zero when $t \rightarrow \infty$. Therefore, we obtain that the following expression as a function of $t$ also tends to 0 when $t \rightarrow \infty$

$$
\sup _{k}\left\|\left(e^{i t H([t])}-e^{i t H_{k}}\right) \phi_{t}\right\| .
$$

Now, let $\varepsilon>0$ be given and choose $T_{0}$ such that $\left\|\left(e^{i t H([t])}-e^{i t H_{k}}\right) \phi_{t}\right\|<\varepsilon / 2$ for $t \geq T_{0}$. Fix $k_{0}$ such that if $t \geq T_{0}$, then $\left\|\left(e^{i t H_{k}}-e^{i t H}\right) \phi_{t}\right\|<\varepsilon / 2$. Then it is straightforward to verify that if $t \geq T_{0}$, then

$$
\left\|\left(e^{i t H([t])}-e^{i t H}\right) \phi_{t}\right\| \leq\left\|\left(e^{i t H([t])}-e^{i t H_{k}}\right) \phi_{t}\right\|+\left\|\left(e^{i t H_{k}}-e^{i t H}\right) \phi_{t}\right\|
$$

so that this latter inequality is less than $\varepsilon$. Moreover,

$$
\left\|\left(e^{i t H([t])} \phi_{t}-\Omega_{+}\right) \phi\right\| \leq\left\|\left(e^{i t H([t])}-e^{i t H}\right) \phi_{t}\right\|+\left\|\left(e^{i t H} \phi_{t}-\Omega_{+}\right) \phi\right\| .
$$

Then it follows that the left-hand side of (14) can be made arbitrarily small. Finally, from the above estimates we obtain the existence of $W_{ \pm}$. Furthermore $\Omega_{ \pm}=$ $W_{ \pm}$.

Now, the next corollary is an immediate consequence of the foregoing theorem.

Corollary 3. Suppose $\Omega_{ \pm}\left(H_{n}, H_{0}\right)=s-\lim _{t \rightarrow \pm \infty} e^{i t H_{n}} e^{-i t H_{0}}$ exist, and let $W(t)$ $=e^{i t H([t])} e^{-i t H_{0}}$. Then the discontinuous dynamics $W(t)$ can be approximated in the strong sense by the sequence $e^{i t H_{n}} e^{-i t H_{0}}$. Moreover,

$$
W_{ \pm}=s-\lim _{n \rightarrow \infty} \Omega_{ \pm}\left(H_{n}, H_{0}\right) .
$$

Corollary 4. Let $R(\zeta, H)$ be the resolvent of the self-adjoint operator $H$. Then

(a) $H W_{ \pm}=W_{ \pm} H_{0}$.

(b) $s-\lim _{t \rightarrow \pm \infty} R\left(\xi, H([t]) W_{ \pm}=W_{ \pm} R\left(\xi, H_{0}\right)\right.$.

(c) Let $D(H([t]))$ be the domain of $H([t])$. Then, $W_{ \pm}(D(H([t])))$ is contained in $D\left(H_{0}\right)$.

(d) $e^{-i t H} W_{ \pm}=W_{ \pm} e^{-i t H_{0}}$.

Proof. (a) follows directly, since $W_{ \pm}=\Omega_{ \pm}$. On the other hand it is clear that $s-\lim _{t \rightarrow \infty} R(\xi, H([t]))=R(\xi, H)$. Then (b) is obtained. In order to prove (c) we recall that $D(H)=D\left(H_{0}\right)=D\left(H_{n}\right)$ for all $n$.

\section{Applications}

In this section we show the existence of the impulsive wave operators $W_{ \pm}$defined by equation (9) when the free dynamics is generated by the Laplacian $-\Delta$ on $L^{2}\left(\mathbb{R}^{3}\right)$ and the impulsive dynamics is defined by $e^{i t H([t])}$, where $H(t)=-\Delta+V(t, x)$ and $V(t, x)$ denotes the operator of multiplication by a function $V(t, x), x \in \mathbb{R}^{3}$. The main assumptions needed are given by a mild restriction on the convergence of the series whose general term is defined by the jump operator associated to the function $F(t)=e^{i t H([t])} e^{-i t H}$.

Let us begin with the following lemma. 
Lemma 5. Let $H([t])=H_{0}+V([t])$ be self-adjoint, and suppose that $V([t])=$ $V_{2}([t])+V_{r}([t])$ where $V_{2}([t]) \in L^{2}\left(\mathbb{R}^{3}\right)$ and $V_{r}([t]) \in L^{r}\left(\mathbb{R}^{3}\right)$ for all $t$ and $2 \leq$ $r<3$. Let us assume the existence of positive constants $\alpha, \beta$ such that $\alpha<\frac{1}{2}$ and $2 \leq r(1+\beta)<3$. Then for all $\varphi$ belonging to the Schwartz space $\mathcal{S}$ the function

$$
f(t)=\left\|V([t]) e^{-i t H_{0}} \varphi\right\|_{2}
$$

is in $L^{1}([1,+\infty))$.

Proof. Let us recall that the dynamics generated by $H_{0}=-\Delta$ satisfies

$$
\left\|e^{-i t H_{0}} \varphi\right\|_{q} \leq|t|^{-3 / 2+3 / p}\|\varphi\|_{q}
$$

whenever $1 / p+1 / q=1$, and $2 \leq p \leq \infty$ (see e.g. [8]). Now, let us choose $p$ such that $1 / p+1 / r=1 / 2$. Then by Hölder's inequality we obtain

$$
\begin{aligned}
\|V([t])\|_{2}\left\|e^{-i t H_{0}} \varphi\right\|_{2} & \leq\left\|V_{2}([t])\right\|_{2}\left\|e^{-i t H_{0}} \varphi\right\|_{\infty}+\left\|V_{r}([t])\right\|\left\|_{r}\right\| e^{-i t H_{0}} \varphi \|_{q} \\
& \leq C_{1}|t|^{\alpha-3 / 2}\|\varphi\|_{1}+C_{2}|t|^{\beta-3 / 2+3 / p}\|\varphi\|_{q} .
\end{aligned}
$$

Since $1 / r=1 / 2-1 / p$, then $\beta<1 / 2-3 / p$. Thus $f$ is in $L^{1}([1, \infty))$.

2.1. Remark. We notice that under the assumptions of Lemma 5 the $L^{2}$ norm of the function $V(t, x)$ is of exponential growth with respect to the $t$ variable.

Let us denote by $L_{y}, y \in \mathbb{R}^{3}$, the translation operator acting on $L^{2}\left(\mathbb{R}^{3}\right)$ defined as $\left(L_{y} \phi\right)(x)=\phi(x-y)$.

Proposition 6. Let $H([t])=H_{0}+V([t])$ be as above, where $V([t])$ verifies the hypothesis of Lemma 5, and let $J_{k}$ be the jump operator of the function $e^{i t H([t])}$ at the integer $k$. Let $\varphi \in L^{2}\left(\mathbb{R}^{3}\right)$, and suppose that

$$
\int_{-\infty}^{+\infty}\left\|J_{k} L_{y} \varphi\right\|_{2} d y \leq C k^{\lambda}
$$

for some positive constant $C$ and $\lambda<1 / 2$. Then $W_{ \pm}=s-\lim _{t \rightarrow \pm \infty} e^{i t H([t])} e^{-i t H_{0}}$ exist.

Proof. Let $\varphi$ be a Schwartz function and define $h(t, s)$ by the following $\mathcal{H}$-valued function:

$$
h(t,[s])=e^{i t H([s])} e^{-i t H_{0}} \varphi .
$$

Thus,

$$
D_{1} h(t, s)=e^{i t H([s])} V([s]) e^{-i t H_{0}} \varphi .
$$

On the other hand, we notice that $\left\|D_{1} h(s,[s])\right\|_{2}=\left\|V([s]) e^{-i s H_{0}} \varphi\right\|_{2}$. Moreover the function $f(s)=\left\|V([s]) e^{-i s H_{0}} \varphi\right\|_{2}$ is in $L^{1}([1, \infty))$, as follows from the previous Lemma 5 .

Next, we claim that the real function $M(t)$ defined by $M(t)=\|h(t,[t])\|_{2}$ verifies the hypothesis of Lemma 1 . To this end we recall the integral representation of the free dynamics

$$
e^{-i k H_{0}} \varphi=\frac{1}{(4 i \pi k)^{3 / 2}} \int_{\mathbb{R}^{3}} e^{-i\langle y, y\rangle(4 i k)^{-1}} L_{y} \varphi d y .
$$

From this last identity we get

$$
e^{i k H(k)}\left(e^{-i k H_{0}} \varphi\right)=\frac{1}{(4 i \pi k)^{3 / 2}} \int_{\mathbb{R}^{3}} e^{-i\langle y, y\rangle(4 i k)^{-1}} e^{i k H(k)} L_{y} \varphi d y .
$$


Furthermore, noting that $J_{k}=e^{i k H(k)}-e^{i k H(k-1)}$,

$$
\left\|\left(e^{i k H(k)}-e^{i k H(k-1)}\right) e^{-i k H_{0}} \varphi\right\|_{2}=\frac{1}{\left|(4 \pi k)^{3 / 2}\right|}\left\|\int_{\mathbb{R}^{3}} e^{-i\langle y, y\rangle(4 i k)^{-1}} J_{k} L_{y} \varphi d y\right\|_{2} .
$$

Then we finally obtain

$$
\left\|\left(e^{i k H(k)}-e^{i k H(k-1)}\right) e^{-i k H_{0}} \varphi\right\|_{2} \leq \frac{1}{\left|(4 \pi k)^{3 / 2}\right|} \int_{\mathbb{R}^{3}}\left\|J_{k} L_{y} \varphi\right\|_{2} d y .
$$

Hence, for a given $\varphi$, by hypothesis we get that there exists a constant $C_{\varphi}$ such that

$$
\left\|\left(e^{i k H(k)}-e^{i k H(k-1)}\right) e^{-i k H_{0}} \varphi\right\|_{2} \leq C_{\varphi} k^{\lambda-\frac{3}{2}} .
$$

Since $\lambda<\frac{1}{2}$ this last inequality proves that the series whose general term is the left-hand side of (20) converges. Thus, the hypotheses of Lemma 1 are now indeed verified, hence the impulsive wave $W_{ \pm}$operators exist.

2.2. Remark. We should point out that the above results establish the existence of the wave operators for the next abstract problem. Let $H(t)=H_{0}+V(t)$, for $t \in \mathbb{R}$

$$
\begin{aligned}
& i \frac{\partial u}{\partial t}=H([t]) u, \quad n \leq t<n+1, \\
& \lim _{\varepsilon \rightarrow 0^{+}} u(n+\varepsilon)=u(n), \\
& \lim _{\varepsilon \rightarrow 0^{+}} u(n+1-\varepsilon)=e^{i(n+1) H_{n}}, \quad n=0, \pm 1, \pm 2, \ldots .
\end{aligned}
$$

Then, the solutions of the perturbed equation (21) are compared asymptotically with those of the free equation

$$
i \frac{\partial u}{\partial t}=H_{0} u
$$

The next example had been considered by Cortés et al. [2], in connection with impulsive linear differential equations [1]. Let $U(t)=e^{-i t H}$ be a one-parameter group of operators acting on a Hilbert space $\mathcal{H}$, and let $\left\{C_{[t]}, t \in \mathbb{R}\right\}$ be a family of contraction operators on $\mathcal{H}$. Then define

$$
\Lambda(t)=\prod_{k=[t]}^{1} U^{*}(k) C_{k} U(k) .
$$

We should remark that the authors of [2] impose the operators $C_{k}$ to be unitary, we notice that this hypothesis can be removed completely and assume instead the mild restrictions given below. Let $\phi \in \mathcal{H}$ and define $h(t,[t])=\Lambda(t) e^{-i t H_{0}} \phi$. Suppose that:

1. $\int_{0}^{\infty}\left\|C_{1} U(1) e^{-i t H_{0}} \phi\right\| d t<\infty$ for all $\phi$ belonging to a dense subspace $\mathcal{D}$ contained in $\operatorname{Dom}(H)$.

2. $\sum_{k=0}^{\infty}\|J(k) \phi\|<\infty$, for all $\phi \in \mathcal{D}$ where $J(k)$ is the jump operator defined by equation (10).

Then it is straightforward to verify under hypotheses (1) and (2) above that the conditions of Lemma 1 are satisfied by the $H$-valued function $t \rightarrow h(t,[t])$. Therefore, it follows that the impulsive wave operators $W_{ \pm}=s-\lim _{t \rightarrow \pm \infty} \Lambda(t) e^{-i t H_{0}}$ exist in this case. 


\section{REFERENCES}

[1] D. D. Bainov and N. V. Milev, Stability of linear impulsive differential equations, Int. J. Systems Sci., Vol. 21, 11 (1990), 2217-2224. MR 91k:34067

[2] V. Cortés, C. Fernández, and R. Rebolledo, Wave operators for discontinuous dynamics, submitted.

[3] V. Cortés and H. Prado, Weak scattering and impulsive systems, Preprint.

[4] J. Cook, Cargase Lectures in Theoretical Physics, (F. Lurchit, ed.) Gordon and Breach, New York, 1967.

[5] E. B. Davies, Time dependent scattering theory, Math. Ann. 210 (1974), 149-162. MR 50:1028

[6] J. S. Howland, Stationary scattering theory for time dependent Hamiltonian, Math. Ann. 207 (1974), 315-335. MR 49:11284

[7] E. J. P. Schmidt, On scattering by time dependent perturbations, Indiana Univ. Math. J., Vol. 24, 10 (1975), 925-935. MR 52:1360

[8] M. Reed and B. Simon, Scattering Theory, Vol. 3, Academic Press, New York, 1979.

Departamento de Matemáticas, Universidad Católica del Norte, Casilla 1280, AntoFagasta, Chile

Current address: Departamento de Matemática y Cs. C., Universidad de Santiago de Chile, Casilla 307, Correo 2, Santiago, Chile

E-mail address: hprado@fermat.usach.cl

Facultad de Matemáticas, Pontificia Universidad Católica de Chile, Casilla 114-D, Santiago, Chile 